\title{
The Postoperative Lymphocyte to Monocyte Ratio Change Predicts Poor Clinical Outcome in Patients with Esophageal Squamous Cell Carcinoma Undergoing Curative Resection
}

\author{
Qian Song $\mathbb{D}^{1},{ }^{1}$ Jun-zhou Wu $\mathbb{D},{ }^{2}$ Hui-fen Jiang $\mathbb{D}^{1},{ }^{1}$ Sheng Wang $\mathbb{D},{ }^{1}$ and Shu-nv Cai $\mathbb{D}^{3}$ \\ ${ }^{1}$ Department of Clinical Laboratory, Zhejiang Cancer Hospital, Institute of Cancer and Basic Medicine (ICBM), Chinese Academy of \\ Sciences; Department of Clinical Laboratory, Cancer Hospital of the University of Chinese Academy of Sciences, Hangzhou, \\ Zhejiang, China \\ ${ }^{2}$ Zhejiang Cancer Hospital, Institute of Cancer and Basic Medicine (ICBM), Chinese Academy of Sciences; Cancer Research Institute, \\ Cancer Hospital of the University of Chinese Academy of Sciences; Cancer Research Institute, Hangzhou, Zhejiang, China \\ ${ }^{3}$ Department of Anesthesiology, Zhejiang Cancer Hospital, Institute of Cancer and Basic Medicine (ICBM), Chinese Academy of \\ Sciences; Department of Anesthesiology, Cancer Hospital of the University of Chinese Academy of Sciences, Hangzhou, \\ Zhejiang, China
}

Correspondence should be addressed to Shu-nv Cai; caisn@zjcc.org.cn

Received 11 August 2019; Revised 27 October 2019; Accepted 29 November 2019; Published 22 April 2020

Academic Editor: Paul Ashwood

Copyright (C) 2020 Qian Song et al. This is an open access article distributed under the Creative Commons Attribution License, which permits unrestricted use, distribution, and reproduction in any medium, provided the original work is properly cited.

\begin{abstract}
Background. Postoperative lymphocyte to monocyte ratio (post-LMR) change (LMRc) reflects the dynamic change of balance between inflammatory reaction and immune reaction after curative operation. An elevated preoperative LMR (pre-LMR) has been shown to be a prognostic factor in patients with esophageal squamous cell carcinoma (ESCC), but the clinical value of the LMRc remains unknown. Methods. 674 patients in ESCC undergoing curative operation were enrolled in this study. LMRc $(\mathrm{LMRc}=$ pre-LMR - post-LMR $)$ was counted on the basis of data within one week before and after operation. The median of LMRc was chosen to be the optimal cut-off value to evaluate the prognostic value of LMRc. Results. Kaplan-Meier curves revealed that $L M R c \leq 1.59$ was significantly associated with worse overall survival (OS) $(P=0.003)$ and disease-free survival (DFS) $(P=0.008)$. Multivariate analysis suggested that LMRc could serve as an independent prognostic predictor for both OS $(P=0.006, \mathrm{HR}=0.687,95 \% \mathrm{CI} 0.526-0.898)$ and DFS $(P=0.003, \mathrm{HR}=0.640,95 \%$ CI $0.476-0.859)$. Conclusions. LMRc is a promising prognostic predictor for predicting the worse clinical outcome in patients with ESCC undergoing curative operation.
\end{abstract}

\section{Background}

The incidence of esophageal cancer is increasing, with an estimated 572,000 new cases globally in 2018. In China, esophageal carcinoma is the fifth most common carcinoma and the fourth leading cause of carcinoma mortality [1]. Esophageal squamous cell carcinoma (ESCC) accounts for $90 \%$ of all cases in China $[2,3]$. Despite intensive study aimed at developing therapies, the overall prognosis of patients including those with curative resection remains poor $[4,5]$. Further studies are needed to identify new prognostic or predictive biomarkers that could help stratify patients for treatment.
Systemic inflammation plays a striking part in cancer development and progression [6]. Plentiful studies report that inflammatory biomarkers, for example, neutrophil to lymphocyte ratio (NLR), lymphocyte to monocyte ratio (LMR), and platelet to lymphocyte ratio (PLR), could be an independent prognostic factor in various cancers [7-11].

The treatment such as surgery and chemotherapy could cause change; therefore, the change of inflammatory biomarkers has been paid attention to recently. The dynamic change of NLR could be a better prognostic predictor in several cancers including gastric cancer [12], lung cancer [13], and kidney cancer [14]. Change in LMR could predict the efficacy of chemotherapy in advanced non-small-cell 
lung cancer [15]. The change of PLR was an independent prognostic predictor for clinical outcome in patients with hepatocellular carcinoma [16]. However, whether the dynamic change of systemic inflammatory biomarkers in patients with ESCC is associated with clinical outcome remains unclear. Therefore, the purpose of this study is to explore the relationship between the clinical outcome of ESCC and the dynamic change of systemic inflammatory response, including the change of neutrophil to lymphocyte ratio (NLRc), the change of lymphocyte to monocyte ratio (LMRc), and the change of platelet to lymphocyte ratio (PLRc). The present study is aimed at evaluating whether LMRc has a significant relationship with overall survival (OS) and disease-free survival (DFS) in patients with ESCC.

\section{Methods}

2.1. Patient Selection. We retrospectively analyzed the clinical data of consecutive patients with ESCC at the Cancer Hospital of the University of Chinese Academy of Sciences, Hangzhou, China, which had received curative resection between Feb. 2008 and Feb. 2015. The eligibility criteria included histologically confirmed resectable ESCC. Individuals were excluded from the present study if they have received chemotherapy or radiotherapy before the surgery. The laboratory data, including preoperative neutrophil, lymphocyte, monocyte, and blood platelet, was obtained by preoperative examination one week before surgery and postoperative evaluation one week after surgery. As a result, 674 patients with newly diagnosed ESCC were enrolled in the present research. Follow-up was performed until Aug. 2016. Our research was approved by the Ethics Committee of the Cancer Hospital of the University of Chinese Academy of Sciences. Informed consent was obtained from all individuals.

2.2. Statistical Analysis. The endpoints of this study were OS, which was calculated from the time of diagnosis to the time of any cause of death and DFS that was between diagnosis and occurrence of local recurrence or distant metastases. NLRc $($ NLRc $=$ pre-NLR - post $-N L R)$, LMRc $(L M R c=$ preLMR - post-LMR), and PLRc (PLRc = pre-PLR - post-PLR) were analyzed as continuous variables, which are expressed as median and interquartile range. All clinical features were counted as categorical variables, which are presented as numbers and percentage. Chi-square tests were used to evaluate the relationship between LMRc and clinical features in patients with ESCC. The median of NLRc, LMRc, and PLRc were chosen to be the optimal cut-off value. OS and DFS were calculated using Kaplan-Meier curves and analyzed by the log-rank test. The Kaplan-Meier curve and the log-rank test were analyzed by GraphPad Prism 7 software. The hazard ratio, 95\% confidence interval, and $P$ value were estimated using COX regression analyses. Statistical analyses were performed using the SPSS, version 19.0, statistical software. All the statistical analysis was two sided, and $P<0.05$ was regarded as statistical significance.

\section{Results}

3.1. Patient Characteristics. We enrolled 674 patients with ESCC who had received curative resection between Feb. 2008 and Feb. 2015. The median age of all individuals was 61 years (range: $35-81$ years). The patients with less than 60 years were $310(46.0 \%)$, and the patients with more than 60 years were $364(54.0 \%) .576(85.5 \%)$ cases were male, and $98(14.5 \%)$ cases were female. There were 115 (17.1\%) with stage $1 \mathrm{a}-1 \mathrm{~b}, 226$ (33.5\%) with stage $2 \mathrm{a}-2 \mathrm{~b}$, and $333(49.4 \%)$ with stage $3 a-3 c$. There were $293(43.5 \%)$ cases without lymph node metastasis, and 381 (56.5\%) cases with lymph node metastasis. There were $468(69.4 \%)$ patients without vessel invasion, and $206(30.6 \%)$ patients with vessel invasion. 251 (37.2\%) patients have nerve infiltration, while 423 $(62.8 \%)$ patients have no nerve infiltration. Table 1 summarizes the clinical features of all patients in ESCC undergoing curative surgical resection, which was, in some cases, followed by adjuvant therapies including chemotherapy and radiotherapy.

3.2. Differences in OS and DFS according to LMRc. We chose the median as the cut-off value of -3.02 for NLRc, 1.59 for LMRc, and -104.89 for PLRc. We found that patients with lower LMRc $(\mathrm{LMRc} \leq 1.59)$ were more likely to be male $(P<0.001)$, have increased hospital time $(\geq 14$ days $)$ $(P=0.002)$ and lower dynamic change for NLR $(P<0.001)$, LMR $(P<0.001)$, and PLR $(P=0.003)$. Lower LMRc $(\mathrm{LMRc} \leq 1.59)$ predicts worse clinical outcomes for OS $(P=0.003)$ and DFS $(P=0.008)$ (Figure 1$)$. Lower NLRc and lower PLRc have no significant relationship with OS and DFS (data not shown). Spearman's analysis showed that LMRc were correlated with sex $(P<0.001)$ and hospital time $(P=0.019)$ (Table 2).

Univariate analysis indicated lower LMRc, pathology grade, depth of tumor, lymph node metastasis, pathological stage, vessel invasive, and nerve infiltration as poor prognostic factors for OS (Table 3). In the multivariate analysis, we identified LMRc $(P=0.006)$, pathology grade $(P=0.020)$, lymph node metastasis $(P<0.001)$, and nerve infiltration $(P=0.002)$ as independent prognostic factors for OS (Table 3).

For DFS, in the multivariate analysis that included LMRc, pathology grade, lymph node metastasis, pathological stage, nerve infiltration, and treatment regimen proven to be significant factors in a univariate analysis, we found that LMRc, pathology grade, lymph node metastasis, nerve infiltration, and treatment regimen could be independent prognostic predictors (for LMRc: $\mathrm{HR}=0.640$; 95\% CI 0.476-0.859; $P=0.003$; Table 4).

\section{Discussion}

In the present study, we demonstrated for the first time that lower LMRc $(\mathrm{LMRc} \leq 1.59)$ represents a novel independent poor prognostic biomarker in patients with ESCC undergoing curative resection. Accumulating studies have demonstrated that systemic inflammatory response, particularly NLR, LMR, and PLR, could be an independent prognostic 
TABLE 1: Demographic and clinical data of 674 ESCC patients according to LMR change.

\begin{tabular}{|c|c|c|c|c|}
\hline \multirow{2}{*}{ Characteristics } & \multirow{2}{*}{$N=674(\%)$} & \multicolumn{2}{|c|}{ LMRc } & \multirow{2}{*}{$P$ value } \\
\hline & & $\leq 1.59(N=337)(\%)$ & $>1.59(N=337)(\%)$ & \\
\hline \multicolumn{5}{|l|}{ Sex } \\
\hline Male & $576(85.5)$ & $307(91.1)$ & $269(79.8)$ & \multirow{2}{*}{$<0.001$} \\
\hline Female & $98(14.5)$ & $30(8.9)$ & $68(20.2)$ & \\
\hline \multicolumn{5}{|l|}{ Age } \\
\hline$\leq 60$ years & $310(46.0)$ & $146(43.3)$ & $164(48.7)$ & \multirow{2}{*}{0.164} \\
\hline$>60$ years & $364(54.0)$ & $191(56.7)$ & $173(51.3)$ & \\
\hline \multicolumn{5}{|l|}{ Pathology grade } \\
\hline Well & $49(7.4)$ & $23(7.0)$ & $26(7.8)$ & \multirow{4}{*}{0.304} \\
\hline Middle & $446(67.5)$ & $231(70.0)$ & $215(65.0)$ & \\
\hline Poorly & $164(24.8)$ & $76(23.0)$ & $88(26.6)$ & \\
\hline Undifferentiated & $2(0.3)$ & $0(0)$ & $2(0.6)$ & \\
\hline \multicolumn{5}{|l|}{ Depth of tumor } \\
\hline T1a-1b & $64(9.5)$ & $29(8.6)$ & $35(10.4)$ & \multirow{3}{*}{0.670} \\
\hline $\mathrm{T} 2$ & $129(19.1)$ & $63(18.7)$ & $66(19.6)$ & \\
\hline $\mathrm{T} 3$ & $481(71.4)$ & $245(72.7)$ & $236(70.0)$ & \\
\hline \multicolumn{5}{|l|}{ Lymph node metastasis } \\
\hline No & $293(43.5)$ & $150(44.5)$ & $143(42.4)$ & \multirow{4}{*}{0.142} \\
\hline N1 & $212(31.5)$ & $101(30.0)$ & $111(32.9)$ & \\
\hline $\mathrm{N} 2$ & $117(17.4)$ & $53(15.7)$ & $64(19.0)$ & \\
\hline N3 & $52(7.7)$ & $33(9.8)$ & $19(5.6)$ & \\
\hline \multicolumn{5}{|l|}{ Pathological stage } \\
\hline $1 a-1 b$ & $115(17.1)$ & $56(16.6)$ & $59(17.5)$ & \multirow{3}{*}{0.949} \\
\hline $2 a-2 b$ & $226(33.5)$ & $113(33.5)$ & $113(33.5)$ & \\
\hline $3 a-3 c$ & $333(49.4)$ & $168(49.9)$ & $165(49.0)$ & \\
\hline \multicolumn{5}{|l|}{ Vessel invasive } \\
\hline Yes & $206(30.6)$ & $101(30.0)$ & $105(31.2)$ & \multirow{2}{*}{0.738} \\
\hline No & $468(69.4)$ & $236(70.0)$ & $232(68.8)$ & \\
\hline \multicolumn{5}{|l|}{ Nerve infiltration } \\
\hline Yes & $251(37.2)$ & $125(37.1)$ & $126(37.4)$ & \multirow{2}{*}{0.936} \\
\hline No & $423(62.8)$ & $212(62.9)$ & $211(62.6)$ & \\
\hline \multicolumn{5}{|l|}{ Treatment regimen } \\
\hline S & $457(67.8)$ & $224(66.5)$ & $233(69.1)$ & \multirow{3}{*}{0.757} \\
\hline S+postoperative C & $155(23.0)$ & $81(24.0)$ & $74(22.0)$ & \\
\hline S+postoperative CRT & $62(9.2)$ & $32(9.5)$ & $30(8.9)$ & \\
\hline \multicolumn{5}{|l|}{ Hospital time } \\
\hline$\leq 14$ days & $560(83.1)$ & $265(78.6)$ & $295(87.5)$ & \multirow{2}{*}{0.002} \\
\hline$>14$ days & $114(16.9)$ & $72(21.4)$ & $42(12.5)$ & \\
\hline NLRc & & & & \\
\hline Median & $-3.02(-4.64--1.69)$ & $-2.26(-3.93--0.95)$ & $-3.78(-5.39--2.49)$ & $<0.001$ \\
\hline LMRc & & & & \\
\hline Median & $1.59(0.71-2.69)$ & $0.71(0.05-1.19)$ & $2.68(2.02-3.54)$ & $<0.001$ \\
\hline PLRc & & & & \\
\hline Median & $-104.89(-162.16--54.85)$ & $-100.65(-159.19--38.38)$ & $-108.64(-163.21--72.47)$ & 0.003 \\
\hline
\end{tabular}

predictor in a variety of carcinomas including ESCC $[9,17$, 18]. The dynamic change of systemic inflammatory biomarkers reflects the change between the inflammation response and immune response in patients after treatments.
Recently, some studies concentrated on the relationship between the dynamic change of systemic response and clinical outcome in patients after therapies. Postoperative elevation of NLR predicts poor clinical outcome in some cancers 


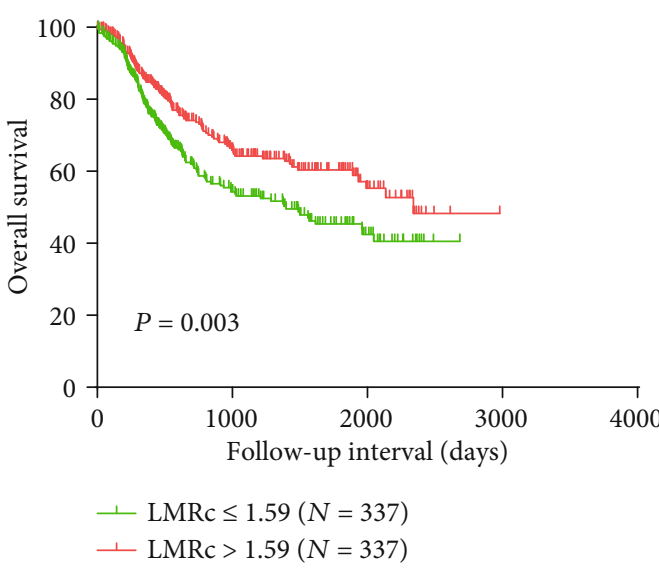

(a)

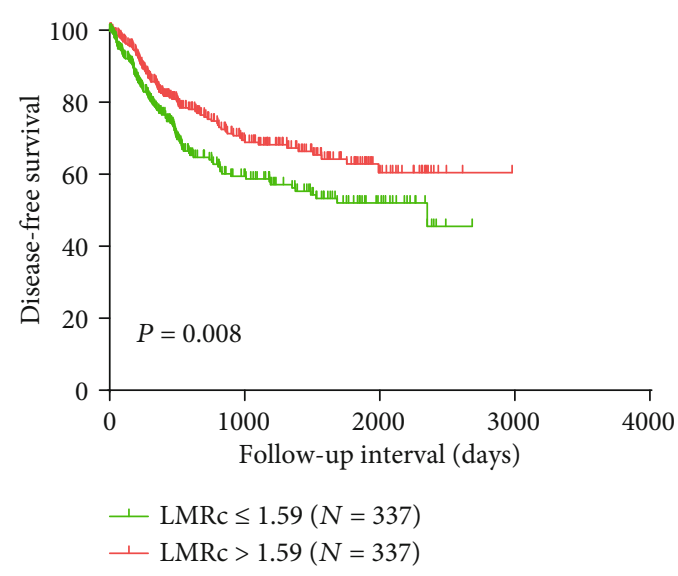

(b)

Figure 1: Overall survival and disease-free survival analysis according the LMRc (a, b).

TABLE 2: Spearman's analysis of correlation between LMR change and clinicopathological features.

\begin{tabular}{lcc}
\hline Variables & \multicolumn{1}{c}{ LMRc } \\
\hline Sex & Spearman correlation & $P$ value \\
Age (years) & -0.198 & $<\mathbf{0 . 0 0 1}$ \\
Pathology grade & -0.056 & 0.144 \\
Depth of tumor & 0.019 & 0.619 \\
Lymph node metastasis & -0.04 & 0.3 \\
Pathological stage & -0.009 & 0.811 \\
Vessel invasive & -0.02 & 0.604 \\
Nerve infiltration & 0.014 & 0.725 \\
Treatment regimen & -0.004 & 0.91 \\
Hospital time (days) & -0.041 & 0.291 \\
\hline
\end{tabular}

such as gastric cancer [12], lung cancer [13], and kidney cancer [14]. A retrospective study with 65 patients following esophageal resection proved that a higher NLRc predicts complications [19]. LMRc represents the prognostic factor in patients with lung cancer who received chemotherapy [15]. PLRc was associated with prognosis in patients with hepatocellular carcinoma [16]. To the best of our knowledge, in patients with ESCC, up to now, a potential prognostic value has not been investigated. Therefore, we evaluated the prognostic significance of NLRc, LMRc, and PLRc regarding two different endpoints.

We found that lower LMRc $(L M R c \leq 1.59)$ was significantly associated with gender (male) and hospital time ( $\geq 14$ days). The incidence and mortality rates in male are 2 -fold to 3 -fold than those in female globally [1]. The eligibility criteria were consecutive patients with newly diagnosed ESCC who received curative surgical resection. There is a possibility that LMRc is related to gender. The relationship between LMRc and hospital time indicated that lower LMRc might be in a bad condition after surgery. Patients with lower LMRc may have complications, including fever or infection. Therefore, lower LMRc had significant longer hospital time after operation.
To date, research has emerged that shows inconsistent results about the prognostic value of microvascular invasion in patients with ESCC [20].The prognostic value of microvascular invasion in ESCC was investigated in various studies, and many suggested microvascular invasion as a crucial prognostic factor in ESCC and is related to adverse prognosis [2124], whereas some did not reach any conclusive results indicating that microvascular invasion is correlated to the clinical outcome of ESCC $[25,26]$. In the present study, we did not find that microvascular invasion could be an independent prognostic indicator. However, LMRc was an independent prognostic predictor for OS and DFS using multivariate analysis. These findings may contribute to explaining why lower LMRc are associated with hospital time ( $\geq 14$ days). In our study, we found that NLRc and PLRc had no significant association with OS or DFS. Future studies about different clinical laboratories and races are needed to prove the findings. This study may impact the treatment practice for ESCC.

Although the molecular mechanism behind this prognostic significance remains hypothetical, published research side with our clinical findings. Monocytes, which constitute about $5 \%$ of the circulating leukocyte pool, play a crucial part in innate immunity [27]. Tumors are infiltrated with immune competent cells reflecting the antitumor response. Tumorassociated macrophages (TAMs), which are derived from monocytes, are recruited to the microenvironment by chemotactic factors [28]. TAMs play a promising role in angiogenesis, invasion, and worse clinical outcome in various cancers [29-31]. Therefore, the absolute monocyte count may represent formation or presence of TAMs. On the one hand, TAMs promote tumor through stimulating the growth of cancer cells, promoting migration and metastasis [32]. Moreover, TAMs, which produce enzymes and inhibitors digesting the extracellular matrix, contribute to tumor invasion and migration $[33,34]$. On the other hand, TAMs suppress the immune response by secreting chemokine that recruit $\mathrm{T}$ cell subsets without cytotoxic function [35]. A growing number of clinical research support the protumor role of TAMs in cancers, showing that TAMs could be an independent prognostic predictor in various cancers [36]. To reinforce the prognostic value of monocytes, we combined them with 
TABLE 3: Overall survival analyses according to LMR change in 674 patients with ESCC.

\begin{tabular}{|c|c|c|c|c|c|c|}
\hline \multirow{2}{*}{ Variables } & \multicolumn{3}{|c|}{ Univariate } & \multicolumn{3}{|c|}{ Multivariate } \\
\hline & $\mathrm{HR}$ & $95 \% \mathrm{CI}$ & $P$ value & HR & $95 \% \mathrm{CI}$ & $P$ value \\
\hline NLRc & 1.013 & $0.972-1.055$ & 0.542 & & & \\
\hline LMRc & 0.670 & $0.515-0.870$ & 0.003 & 0.687 & $0.526-0.898$ & 0.006 \\
\hline PLRc & 1.000 & $0.998-1.001$ & 0.485 & & & \\
\hline Sex & 1.249 & $0.841-1.855$ & 0.270 & & & \\
\hline Age (years) & 1.000 & $0.983-1.017$ & 0.999 & & & \\
\hline Pathology grade & 1.566 & $1.228-1.998$ & $<0.001$ & 1.357 & $1.049-1.757$ & 0.020 \\
\hline Depth of tumor & 1.506 & $1.173-1.933$ & 0.001 & 1.385 & $0.994-1.930$ & 0.055 \\
\hline Lymph node metastasis & 1.738 & $1.528-1.978$ & $<0.001$ & 1.746 & $1.416-2.154$ & $<0.001$ \\
\hline Pathological stage & 1.897 & $1.538-2.342$ & $<0.001$ & 0.811 & $0.555-1.186$ & 0.280 \\
\hline Vessel invasive & 1.770 & $1.356-2.310$ & $<0.001$ & 1.172 & $0.875-1.570$ & 0.287 \\
\hline Nerve infiltration & 1.841 & $1.419-2.390$ & $<0.001$ & 1.535 & $1.164-2.025$ & 0.002 \\
\hline Treatment regimen & 1.012 & $0.9-1.137$ & 0.847 & & & \\
\hline Hospital time (days) & 1.007 & $0.997-1.016$ & 0.169 & & & \\
\hline
\end{tabular}

TABLE 4: Disease-free survival analyses according to LMR change in 674 patients with ESCC.

\begin{tabular}{|c|c|c|c|c|c|c|}
\hline \multirow{2}{*}{ Variables } & \multicolumn{3}{|c|}{ Univariate } & \multicolumn{3}{|c|}{ Multivariate } \\
\hline & HR & $95 \% \mathrm{CI}$ & $P$ value & HR & $95 \% \mathrm{CI}$ & $P$ value \\
\hline$\overline{\text { NLRc }}$ & 1.013 & $0.976-1.060$ & 0.591 & & & \\
\hline LMRc & 0.677 & 0.507-0.905 & 0.008 & 0.640 & $0.476-0.859$ & 0.003 \\
\hline PLRc & 1.000 & $0.998-1.001$ & 0.617 & & & \\
\hline Sex & 1.210 & $0.788-1.858$ & 0.383 & & & \\
\hline Age (years) & 0.992 & $0.973-1.010$ & 0.376 & & & \\
\hline Pathology grade & 1.652 & $1.264-2.157$ & $<0.001$ & 1.515 & $1.140-2.014$ & 0.004 \\
\hline Depth of tumor & 1.144 & $0.902-1.450$ & 0.266 & & & \\
\hline Lymph node metastasis & 1.598 & $1.377-1.853$ & $<0.001$ & 1.534 & $1.222-1.926$ & $<0.001$ \\
\hline Pathological stage & 1.558 & $1.257-1.932$ & $<0.001$ & 0.824 & $0.595-1.140$ & 0.242 \\
\hline Vessel invasive & 1.292 & $0.949-1.760$ & 0.104 & & & \\
\hline Nerve infiltration & 1.623 & $1.213-2.170$ & 0.001 & 1.580 & $1.160-2.150$ & 0.004 \\
\hline Treatment regimen & 1.385 & $1.233-1.556$ & $<0.001$ & 1.284 & $1.133-1.45$ & $<0.001$ \\
\hline Hospital time (days) & 1.000 & 0.989-1.012 & 0.975 & & & \\
\hline
\end{tabular}

lymphocyte. Lymphocytes, particularly cytotoxic lymphocytes, function as the host defense against cancer cells and are applied in immunotherapy $[37,38]$. Lymphocytopenia is a well-known result of a systemic inflammatory response accompanying malignant diseases [37]. The advantage of the present study is the large sample size. However, some shortcomings have to be taken into account, mostly based on the retrospective study and a single-center design.

\section{Conclusions}

To the best of our knowledge, our study is the first report indicating that LMRc is a novel independent prognostic factor in patients with ESCC undergoing curative resection. Large-scale prospective research are needed to prove our findings.

\section{Abbreviations}

post-LMR: Postoperative lymphocyte to monocyte ratio LMRc: Postoperative lymphocyte to monocyte ratio change

pre-LMR: Preoperative lymphocyte to monocyte ratio

ESCC: $\quad$ Esophageal squamous cell carcinoma

OS: Overall survival

DFS: $\quad$ Disease-free survival

NLR: $\quad$ Neutrophil to lymphocyte ratio

LMR: $\quad$ Lymphocyte to monocyte ratio

PLR: $\quad$ Platelet to lymphocyte ratio

NLRc: The change of neutrophil to lymphocyte ratio

PLRc: $\quad$ The change of platelet to lymphocyte ratio

HR: $\quad$ Hazard ratio

95\% CI: $\quad$ 95\% confidence interval

P: $\quad$ Probability. 


\section{Data Availability}

The datasets used in the present research are available from the corresponding author on reasonable request.

\section{Ethical Approval}

All procedures in our research were based on the ethical standards of the World Medical Association Declaration of Helsinki. The present research approval was obtained from the ethics committee at Zhejiang Cancer Hospital.

\section{Consent}

All participants gave written informed consent.

\section{Conflicts of Interest}

The authors declare that they have no competing interests.

\section{Authors' Contributions}

QS designed the study and was a major contributor in writing the manuscript. JW collected material and data and was involved in the statistical interpretation of the data. SW and HJ collected material and data and performed statistical analysis. SC drafted the manuscript. All authors read and approved the final manuscript.

\section{Acknowledgments}

This study was funded by the National Natural Science Foundation of China (contract/grant number: 81602615), General research program of Health Department of Zhejiang Province (contract/grant numbers: 2016KYA40, 2016KYB048, and 2020KY480), Zhejiang Youth Talents Project (contract/grant number: 2019RC026), and Science and Technology Department of Zhejiang Province (contract/grant number: 2018C37130). We thank all the included patients and the investigators, including the clinicians and laboratory technicians in our study.

\section{References}

[1] F. Bray, J. Ferlay, I. Soerjomataram, R. L. Siegel, L. A. Torre, and A. Jemal, "Global cancer statistics 2018: GLOBOCAN estimates of incidence and mortality worldwide for 36 cancers in 185 countries," CA: A Cancer Journal for Clinicians, vol. 68, no. 6, pp. 394-424, 2018.

[2] M. Arnold, M. Laversanne, L. M. Brown, S. S. Devesa, and F. Bray, "Predicting the future burden of esophageal cancer by histological subtype: international trends in incidence up to 2030," The American Journal of Gastroenterology, vol. 112, no. 8, pp. 1247-1255, 2017.

[3] G. D. Tran, X. D. Sun, C. C. Abnet et al., "Prospective study of risk factors for esophageal and gastric cancers in the Linxian general population trial cohort in China," International Journal of Cancer, vol. 113, no. 3, pp. 456-463, 2005.

[4] R. Gertler, H. J. Stein, R. Langer et al., "Long-term outcome of 2920 patients with cancers of the esophagus and esophagogastric junction: evaluation of the New Union Internationale
Contre le Cancer/American joint Cancer Committee staging system," Annals of Surgery, vol. 253, no. 4, pp. 689-698, 2011.

[5] W. H. Allum, S. P. Stenning, J. Bancewicz, P. I. Clark, and R. E. Langley, "Long-term results of a randomized trial of surgery with or without preoperative chemotherapy in esophageal cancer," Journal of Clinical Oncology, vol. 27, no. 30, pp. 50625067, 2009.

[6] C. Lin and J. Zhang, "Inflammasomes in inflammationinduced cancer," Frontiers in Immunology, vol. 8, p. 271, 2017.

[7] Y. Chen, H. Yan, Y. Wang, Y. Shi, and G. Dai, "Significance of baseline and change in neutrophil-to-lymphocyte ratio in predicting prognosis: a retrospective analysis in advanced pancreatic ductal adenocarcinoma," Scientific Reports, vol. 7, no. 1, p. 753, 2017.

[8] Q. T. Huang, L. Zhou, W. J. Zeng et al., "Prognostic significance of neutrophil-to-lymphocyte ratio in ovarian cancer: a systematic review and meta-analysis of observational studies," Cellular Physiology and Biochemistry, vol. 41, no. 6, pp. 24112418, 2017.

[9] G. Hu, G. Liu, J. Y. Ma, and R. J. Hu, "Lymphocyte-to-monocyte ratio in esophageal squamous cell carcinoma prognosis," Clinica Chimica Acta, vol. 486, pp. 44-48, 2018.

[10] X. Z. Huang, W. J. Chen, X. Zhang et al., "An elevated plateletto-lymphocyte ratio predicts poor prognosis and clinicopathological characteristics in patients with colorectal cancer: a meta-analysis," Disease Markers, vol. 2017, Article ID 1053125, 10 pages, 2017.

[11] Z. Mei, L. Shi, B. Wang et al., "Prognostic role of pretreatment blood neutrophil-to-lymphocyte ratio in advanced cancer survivors: a systematic review and meta-analysis of 66 cohort studies," Cancer Treatment Reviews, vol. 58, pp. 1-13, 2017.

[12] K. W. Min, M. J. Kwon, D. H. Kim et al., "Persistent elevation of postoperative neutrophil-to-lymphocyte ratio: a better predictor of survival in gastric cancer than elevated preoperative neutrophil-to-lymphocyte ratio," Scientific Reports, vol. 7, no. 1, p. 13967, 2017.

[13] F. Jin, A. Han, F. Shi, L. Kong, and J. Yu, "The postoperative neutrophil-to-lymphocyte ratio and changes in this ratio predict survival after the complete resection of stage I non-small cell lung cancer," OncoTargets and therapy, vol. 9, pp. 65296537, 2016.

[14] A. J. Templeton, J. J. Knox, X. Lin et al., "Change in neutrophilto-lymphocyte ratio in response to targeted therapy for metastatic renal cell carcinoma as a prognosticator and biomarker of efficacy," European Urology, vol. 70, no. 2, pp. 358-364, 2016.

[15] K. Sekine, S. Kanda, Y. Goto et al., "Change in the lymphocyteto-monocyte ratio is an early surrogate marker of the efficacy of nivolumab monotherapy in advanced non-small-cell lung cancer," Lung Cancer, vol. 124, pp. 179-188, 2018.

[16] W. Peng, C. Li, W. J. Zhu et al., "Prognostic value of the platelet to lymphocyte ratio change in liver cancer," The Journal of Surgical Research, vol. 194, no. 2, pp. 464-470, 2015.

[17] Y. Huang, Y. Sun, P. Peng, S. Zhu, W. Sun, and P. Zhang, "Prognostic and clinicopathologic significance of neutrophilto-lymphocyte ratio in esophageal squamous cell carcinoma: evidence from a meta-analysis," OncoTargets and therapy, vol. 10, pp. 1165-1172, 2017.

[18] Y. Yang, H. Xu, L. Zhou et al., "Platelet to lymphocyte ratio is a predictive marker of prognosis and therapeutic effect of postoperative chemotherapy in non-metastatic esophageal 
squamous cell carcinoma," Clinica Chimica Acta, vol. 479, pp. 160-165, 2018.

[19] P. Vulliamy, S. McCluney, S. Mukherjee, L. Ashby, and T. Amalesh, "Postoperative elevation of the neutrophil: lymphocyte ratio predicts complications following esophageal resection," World Journal of Surgery, vol. 40, no. 6, pp. 13971403, 2016.

[20] G. Ma, J. Zhang, H. Jiang et al., "Microvessel density as a prognostic factor in esophageal squamous cell cancer patients: a meta-analysis," Medicine, vol. 96, no. 29, article e7600, 2017.

[21] S. C. Zhang, S. Hironaka, A. Ohtsu et al., "Computer-assisted analysis of biopsy specimen microvessels predicts the outcome of esophageal cancers treated with chemoradiotherapy," Clinical Cancer Research, vol. 12, no. 6, pp. 1735-1742, 2006.

[22] C. H. Shih, S. Ozawa, N. Ando, M. Ueda, and M. Kitajima, "Vascular endothelial growth factor expression predicts outcome and lymph node metastasis in squamous cell carcinoma of the esophagus," Clinical Cancer Research, vol. 6, no. 3, pp. 1161-1168, 2000.

[23] A. Faried, H. Kimura, L. S. Faried et al., "Expression of carbohydrate antigens in human esophageal squamous cell carcinoma: prognostic application and its diagnostic implications," Annals of Surgical Oncology, vol. 14, no. 2, pp. 960-967, 2007.

[24] J. Y. Choi, K. T. Jang, Y. M. Shim et al., "Prognostic significance of vascular endothelial growth factor expression and microvessel density in esophageal squamous cell carcinoma: comparison with positron emission tomography," Annals of Surgical Oncology, vol. 13, no. 8, pp. 1054-1062, 2006.

[25] S. Y. Ha, S. Y. Yeo, Y. H. Xuan, and S. H. Kim, "The prognostic significance of cancer-associated fibroblasts in esophageal squamous cell carcinoma," PLoS One, vol. 9, no. 6, article e99955, 2014.

[26] M. Sarbia, F. Bittinger, R. Porschen, P. Dutkowski, R. Willers, and H. E. Gabbert, "Tumor vascularization and prognosis in squamous cell carcinomas of the esophagus," Anticancer Research, vol. 16, no. 4A, pp. 2117-2121, 1996.

[27] J. Parkin and B. Cohen, "An overview of the immune system," The Lancet, vol. 357, no. 9270, pp. 1777-1789, 2001.

[28] A. Mantovani, T. Schioppa, C. Porta, P. Allavena, and A. Sica, "Role of tumor-associated macrophages in tumor progression and invasion," Cancer Metastasis Reviews, vol. 25, no. 3, pp. 315-322, 2006.

[29] F. Balkwill, "Cancer and the chemokine network," Nature Reviews Cancer, vol. 4, no. 7, pp. 540-550, 2004.

[30] L. Bingle, N. J. Brown, and C. E. Lewis, "The role of tumourassociated macrophages in tumour progression: implications for new anticancer therapies," The Journal of Pathology, vol. 196, no. 3, pp. 254-265, 2002.

[31] S. Tsutsui, K. Yasuda, K. Suzuki, K. Tahara, H. Higashi, and S. Era, "Macrophage infiltration and its prognostic implications in breast cancer: the relationship with VEGF expression and microvessel density," Oncology Reports, vol. 14, no. 2, pp. 425-431, 2005.

[32] A. Mantovani, P. Allavena, A. Sica, and F. Balkwill, "Cancerrelated inflammation,” Nature, vol. 454, no. 7203, pp. 436444, 2008.

[33] L. M. Coussens, C. L. Tinkle, D. Hanahan, and Z. Werb, "MMP-9 supplied by bone marrow-derived cells contributes to skin carcinogenesis," Cell, vol. 103, no. 3, pp. 481-490, 2000.

[34] M. Locati, U. Deuschle, M. L. Massardi et al., "Analysis of the gene expression profile activated by the $\mathrm{CC}$ chemokine ligand
5/RANTES and by lipopolysaccharide in human monocytes," Journal of Immunology, vol. 168, no. 7, pp. 3557-3562, 2002.

[35] A. Sica, P. Allavena, and A. Mantovani, "Cancer related inflammation: the macrophage connection," Cancer Letters, vol. 267, no. 2, pp. 204-215, 2008.

[36] F. Balkwill, K. A. Charles, and A. Mantovani, "Smoldering and polarized inflammation in the initiation and promotion of malignant disease," Cancer Cell, vol. 7, no. 3, pp. 211-217, 2005.

[37] T. Nazir, A. Islam, M. O. Omer, and M. Mustafa, "Lymphocytopenia; induced by vinorelbine, doxorubicin and cisplatin in human cancer patients," Breast Disease, vol. 35, no. 1, pp. 14, 2015.

[38] L. Martinez-Lostao, A. Anel, and J. Pardo, "How do cytotoxic lymphocytes kill cancer cells?," Clinical Cancer Research, vol. 21, no. 22, pp. 5047-5056, 2015. 